This item was submitted to Loughborough's Research Repository by the author.

Items in Figshare are protected by copyright, with all rights reserved, unless otherwise indicated.

\title{
RoCoF calculation using low-cost hardware in the loop: multi-area Nordic power system
}

PLEASE CITE THE PUBLISHED VERSION

https://doi.org/10.1109/SST49455.2020.9264119

PUBLISHER

IEEE

VERSION

AM (Accepted Manuscript)

\section{PUBLISHER STATEMENT}

(c) 2020 IEEE. Personal use of this material is permitted. Permission from IEEE must be obtained for all other uses, in any current or future media, including reprinting/republishing this material for advertising or promotional purposes, creating new collective works, for resale or redistribution to servers or lists, or reuse of any copyrighted component of this work in other works.

\section{LICENCE}

\section{All Rights Reserved}

\section{REPOSITORY RECORD}

Barrios-Gomez, Jose, Francisco Sanchez, Gianfranco Claudio, F Gonzalez-Longatt, M Acosta, and Danijel Topic. 2020. "Rocof Calculation Using Low-cost Hardware in the Loop: Multi-area Nordic Power System". Loughborough University. https://hdl.handle.net/2134/13388771.v1. 


\section{RoCoF Calculation Using Low-Cost Hardware in the Loop: Multi-area Nordic Power System}

\author{
J.A. Barrios-Gomez, F. Sanchez, \\ Gianfranco Claudio \\ Centre for Renewable Energy Systems \\ Technology - CREST \\ Loughborough University \\ Loughborough, United Kingdom \\ J.A.Barrios-Gomez@lboro.ac.uk
}

\author{
F. Gonzalez-Longatt, M. Acosta \\ Department of Electrical Engineering, \\ Information Technology and \\ Cybernetics \\ University of South-Eastern Norway \\ Porsgrunn, Norway \\ flongatt@flongatt.org
}

\author{
Danijel Topic \\ Faculty of Electrical Engineering, \\ Computer Science and Information \\ Technology \\ University of Osijek \\ Osijek, Croatia \\ danijel.topic@ferit.hr
}

\begin{abstract}
This research paper presents a rate of change of frequency (RoCoF) implementation using low-cost hardware in the loop (HIL) with application to Nordic Power System (NPS). Two methods to calculate the RoCoF are presented: Incremental difference one step and Moving window (MW) or rolling window. HIL) approach is used to obtain natural noise found in the frequency signal obtained from real power devices. The low-cost HIL implementation is based on two Arduinos ${ }^{\circledR}$ in the loop. An Arduino ${ }^{\circledR}$ working as standalone is used to generate an analogue signal representative of one area of the NPS, a second Arduino ${ }^{\circledR}$ in the loop contains the RoCoF calculation methods and filters. As the noise is essential affecting the RoCoF calculation, this paper analyses the effect of including lowpass filters (LPFs). Three cases are analysed: Base Case: no filter and noise signal, Case I: LPF applied to the input frequency signal, Case II: LPF at the frequency signal and LFP at the output of the RoCoF (smoothing). Results demonstrate that RoCoF calculation methods worked correctly in the HIL and using two LPFs clean the signal from the noise produced by the Arduino ${ }^{\circledR}$.
\end{abstract}

Keywords-Derivative, Hardware in the Loop, Low Pass Filter, Nordic Power System, RoCoF.

\section{INTRODUCTION}

Nowadays, power system (PS) frequency and rate of change frequency (RoCoF) are growingly essential concepts for load management, protection and system control [1]. Ideally, it is desired to have error-free and noise data that is available with minimal delay [2]. This is not the case in real $\mathrm{PS}$, because the frequency and RoCoF measurement are sensitive to noise and disturbances in the electrical system. Typically to fix this, it is needed heavy filtering to deliver robust and usable data [3]. The RoCoF is an important indicator to evaluate the stability of electric power networks, and they are also used as input for the control and protection devices that are used in electric power systems (EPS) [4]. It is known that RoCoF in modern electric power networks increases as the inertia of the system decreases; this caused by the integration of wind and solar energy, which are energy sources without inertia [5]. RoCoF is the nearly all widely used anti-islanding protection method [6]. Nevertheless, the safety of the relays based on this scheme is continuously disputed [7], as it is susceptible to network disturbances, causing unwanted trips. The solution to this is to compromise the safety and reliability of the relay, this is not simple labour and is further fiddly when the efficiency of marketable available RoCoF relays varies significantly, even having similar configurations [7],[8].

In this paper, the implementation of two methodologies to calculate the RoCoF in low-cost hardware is carried out. The focus is on the algorithms to calculate the derivative and the noise elimination of the signals using lowpass filters (LPFs); these are implemented in low-cost hardware. The impact of these calculations contributes to the safety with which the relay operates, and it is also possible to see how the disturbances affect the local frequency and the RoCoF in an EPS. The main contribution to the development of this paper is the implementation of the RoCoF calculation implemented in a low-cost HIL and applying LPFs to eliminate noise from the signals.

\section{RATE OF CHANGE OF FREQUENCY (ROCOF)}

Frequency drifts subsequently to the islanding be conditional on active power discordance, inertia among the time instance and island. Therefore, calculating the derivative of frequency, moreover called RoCoF, can be accelerated the islanding detection [9]. The variation of the load and input mechanical power produces a change of frequency. Thanks to this variation posterior to disconnect from the primary grid, a dynamic change in frequency is expected, besides the rate of change of frequency usually can be calculated by the following equation [10], [11]:

$$
\frac{d f(t)}{d t}=-\left[\frac{P_{L}(t)-P_{G}(t)}{2 H S_{G R}} f_{0}\right]
$$

where $P_{L}$ is the power demand, $P_{G}$ is the total active power generation in the PS, $S_{G R}$ is distributed generator rating, $H$ is the total system inertia, $f$ is the system frequency, and $f_{0}$ is the rated frequency. Network incidents may also inflict changes in system frequency, culminating in the inappropriate operation of RoCoF relays [7]. The frequency protection relays are founded on $\mathrm{RoCoF}$ for their respond against incidents in the EPS, and the foundation of the method is the Fourier transform and the study of the zero-crossings. RoCoF relays measure the rate of change of frequency, and after the rate of change of frequency oversteps the preestablished setting, a trip signal is beginning. In respect of response time and to prevent false activations, the response time of the relay will be within 50 and $500 \mathrm{~ms}$, this makes the device more secure [12]. The number of frequency points atop which the frequency change will be determined, define the measurement window, the interlocks need to be considered due to the low voltage since it could obstruct the activity of the frequency relay [7].

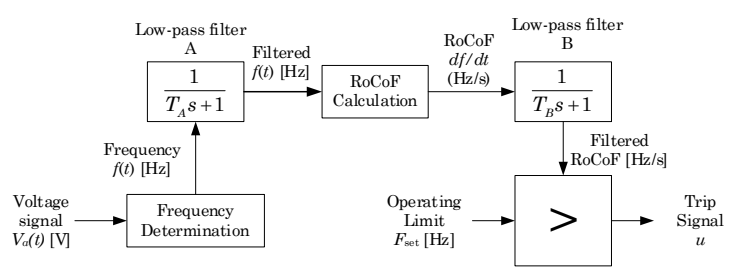

Fig. 1. Typical structure of a generic RoCoF relay. 
The block diagram, of a typical structure of a frequency relay, including the RoCoF action, is shown in Fig. 1. The initial block in the relay model is called frequency determination; It is where the frequency is calculated. Then an anti-aliasing LPF is applied to the frequency; anti-aliasing filter will commonly either allow some aliasing to take place or else reduced some in-band frequencies close to the Nyquist limit. The signal from the filter feeds to a RoCoF calculation block, and the output of the RoCoF block is eventually smoothed by the last LPF, and that respond is compared against value setting and commands trip just after the calculated values surpass pre-set threshold usually within 0.1 and 1.2 Hz/s, these parameters are mentioned in [13], [14].

\section{A. RoCoF definition}

RoCoF function is one of the protective functions used for fast load shedding, to detect loss of grid and to accelerate operation time in under and over frequency conditions. The magnitude of the power imbalance generated from the disturbance defines how fast the system frequency and generator speed changes. The RoCoF is measured in Hertz per second (Hz/s), this might indicate as a measure of the gravity of a disturbance. Disturbances that cause high RoCoF events can have detrimental effects on an EPS. When severe cases exist, the generator protection devices can operate and disconnect the generator from the EPS; synchronous generators can lose synchronism with the EPS, and the RoCoF anti-island relays can operate, disconnecting distributed generation from the PS[15], [16]. Therefore, they could initiate a cascading effect and aggravate the main disturbance, and this could lead to the complete shutdown of the EPS. Consequently, it is essential that EPS are designed to operate in such a way that the severity of high RoCoF events is reduced or avoided [17], [18]. The most representative way of calculating the RoCoF in electrical power systems after a distributing is by using the centre of inertia (definition is presented in [19]). The RoCoF calculated as [20]:

$$
\frac{d f(t)}{d t}=-\Delta P\left(\frac{f_{0}}{2 H S_{G R}}\right)
$$

where $\Delta P$ is the change in power, $f_{0}$ is the system rated frequency, $d f / d t$ is the initial RoCoF, $S_{G R}$ is the rated power of the system and $H$ is the remaining inertia constant of the whole system after the disturbance.

\section{B. Methods to calculate RoCoF}

In this scientific paper, two methods are used to calculate the RoCoF and explaining in the next subsections.

\section{1) Incremental difference one step}

Within the numerical derivatives, there is the method forward difference derivative, which is a simple approximate calculation; therefore it is only carried out the evaluation of the equation (3) for a small, but finite, $t_{i+1}-t_{i}$ [21].

$$
\operatorname{RoCoF}\left(t_{i}\right)=\frac{d f}{d t}=\frac{f\left(t_{i+1}\right)-f\left(t_{i}\right)}{t_{i+1}-t_{i}}
$$

It is also known as the forward difference derivative. Specified the $m(t, f)$ points, it is possible to evaluate $d f / d t$, at $(m-1)$ points applying the equation (3). The forward difference derivative could be converted into a backward difference derivative by changing to a negative value for $t_{i+1}-t_{i}$. This approach can approximate a limit by evaluating what is expected to be close to the limit and producing a difference in the numerator of the expression [22].

\section{2) Moving window or rolling window}

The effects of actual RoCoF measurements on a moving window can be analysed by implementing the following equation:

$$
\operatorname{RoCoF}\left(t_{i}\right)=\frac{d f}{d t}=\frac{f\left(t_{i}\right)-f\left(t_{i}+N\right)}{N T}
$$

where $d f / d t$ is the RoCoF at sample t, $N$ is the number of samples in the moving average window, $T$ is the duration of the moving average window and $f(t)$ is the frequency at sample $t$.

\section{Filter definition}

The function of any filter is to select frequencies within the desired frequency range and to filter out any other frequencies. Thus, using filter techniques is possible to obtain spectra that are free of any ambiguous signals and avoid misinterpretation in the spectra. The essential idea is that the raw signal, which contains noise and the desired information, will pass through a filter, then the filter output contains the desired information. The analog filters use analogue electronic circuits, and the digital filters simply require a digital processor to perform numerical calculations on the sampled values of an analog signal [23]. The advantages of digital filters are as follows: digital filters are programmable, especially they are extremely stable with respect to both time and temperature, a digital filter is much more versatile because signals can be processed in any variety of ways [23], [24].

\section{1) First-order Filter}

LPFs have been used in this research paper, as shown in Fig. 1. An LPF is a filter that accepts signals under cut-off frequency or pass-band and also decreases signals over the cut-off frequency or stop-band. These filters take away part of frequency, generating a smooth response, besides providing small variations in the signal obtained and easy to appreciate the trend by increasing proportions in global signal and noise, with little loss at the output. LPF, mainly moving average filters, also known as Savitzky Golay filters, commonly applied to eliminate unwanted sections from a signal, design decimators and interpolators, perform data averaging, discover patterns, and remove noise. The methodology to create filters infinite impulse response-based, incorporate the Chebyshev, elliptic and Butterworth [23], [25],[26].

As mentioned in RoCoF relay structure, the generated signal from RoCoF calculation is eventually filtered by a function, $\left(T_{a} s+1\right)^{-1}$, create to remove high-frequency transients. Thereby, $T_{a}$ represents the time constant, used for the selected measurement window and time constant of the filters. The result is eventually compared against the threshold value. Thus, when the previous value oversteps the last, it starts a trigger signal. When a trigger decision is performed, the simulation takes it as the operating time. The LPF blocks are shown in Fig. 1 implement the following transfer function:

$$
H(s)=\frac{1}{T_{x} s+1}
$$

where, $s$ is the Laplace operator and $T_{x}$ the time constant of the filter and related to the cut-off frequency. In measurement action, filters are employed the frequency be required to be in to a narrow band, as well as the RoCoF have to not overstep the specific values for the system under study. Consequently, it is usually supposed that RoCoF and frequency and can be determined within $100 \mathrm{~ms}$. 


\section{NORDIC POWER SYSTEM (NPS) MODEL}

The model presented in this research paper is a simplification of the Nordic synchronous system. In Fig 2, the arrows indicate the power flow direction relationship and connections with the countries neighbouring the NPS. The NPS was modelled using one load and one generator by area. The DK2 area does not have hydropower, therefore was modelled as a constant value. The Nordic region possesses an interconnected synchronous system formed of eleven areas. These areas are SE1 to SE4 in Sweden, NO1 to NO5 in Norway, DK2 in Denmark and F1 in Finland. Denmark DK1 belongs to the Nord Pool system but not in the Nordic synchronous area [27]-[29].

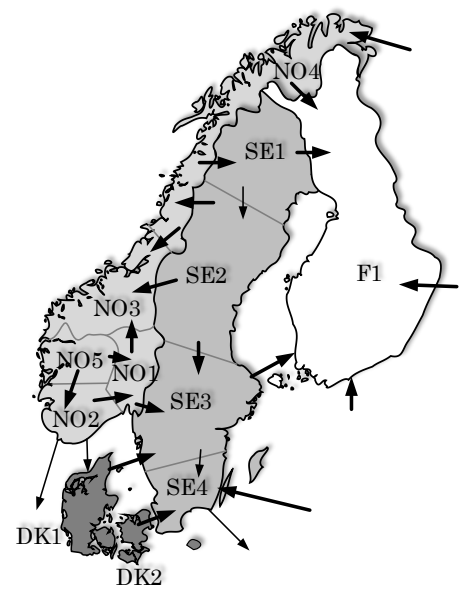

Fig. 2. Map indicating the NPS bidding zones.

For this model, the control structure is a procedure established from a merging of the DC power flow and oscillation equation. Thus, it is possible to expand the system to some arbitrary size. In each area of the electrical system, there are a certain number of energy charges and generating units. The system works at $50 \mathrm{~Hz}$ frequency, and the per-unit method is used in all calculations. Therefore, when the system frequency is $50 \mathrm{~Hz}$ the velocity will be zero, and working in steady-state. The apparent power of the system $\left(S_{\text {base }}\right)$ is 1,000 MVA; there is a global base value. The areas have local bases, associated with the power of the generators. Each modelled area have to the output the generator voltage angles. The turbine governors carry out the primary control in a PS, and the proportional integral derivative (PID) controllers are used to the governing systems. In Fig. 3 shows the block diagram used in this research paper. More details can be seen in [29].

The controller uses the speed change fixed point obtained by the AGC, $\Delta P_{\text {ref }}$, the difference in reference speed $\omega_{\text {ref }}$ and the system speed, $\Delta \omega$, to the input. The integral time constant, $T_{i}$ and proportional gain $K_{p}$ is used to control the signal in the valve position of the turbine $\Delta c$ [24]. The derivative of the PID controller is $K_{\mathrm{D}}$, in the model used in this paper was set to zero, as it was also used in [29].

During a power deviation produced by a disturbance, the controller is incorporated to make certain that the power production is the same as the power demand after the disturbance occurs. The deviation from planned power is calculated and is used as input to the PID integrator block. The droop rho $(\rho)$ is the steady-state feedback which multiplies the deviation in the mechanical power, $\Delta P_{\mathrm{m}}$, through a feedback loop.
The frequency is not an input to the turbine governor, hence does not possible that integrator recover to the reference value. To prevent generators in the system from operating affecting each other is to employ the droop feedback loop. Lastly, the water start-up time of the turbine is $T_{\mathrm{w}}$.

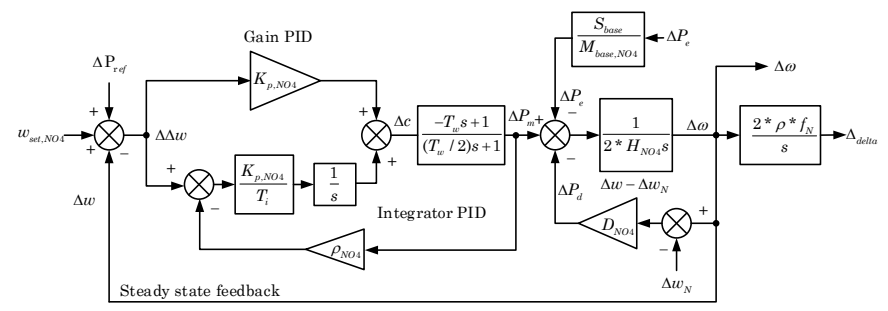

Fig. 3. Block diagram of the NPS. Details of Finland (F1) control area 5.

The block diagram of the turbine governor, turbine and the generator in area F1 in Finland, are shown in Fig. 3, the rest generator subsystems are identical, the difference lies in parameter values which depends on each specific area.

\section{HARDWARE-IN-THE-LOOP IMPLEMENTATION}

Matlab $^{\mathrm{TM}}$ has the Simulink ${ }^{\circledR}$ support package for Arduino ${ }^{\circledR}$ hardware; This can be used to develop and simulate algorithms that run independently on Arduino ${ }^{\circledR}$. It also has simultaneous link blocks for configuring and accessing the Arduino ${ }^{\circledR}$ inputs and outputs and external mode for setting interactive parameters and monitoring the signal while the algorithm runs on the device [30].

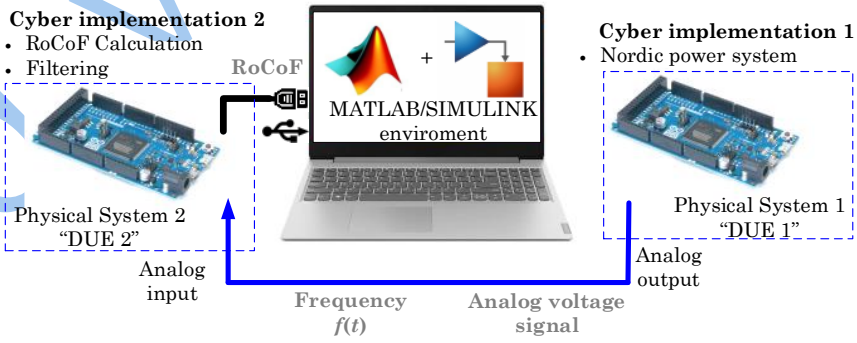

Fig. 4. HIL structure for cyber-physical simulation.

A structure for the implementation of RoCoF using a lowcost HIL applied to the NPS model is shown in Fig. 4. In this scheme DUE1 is in standalone and contains the NPS of 11 areas, it outputs an analogue signal with the frequency. On the other hand, DUE2 receives the frequency signal and performs RoCoF calculations, which are displayed on the computer.

Arduino ${ }^{\circledR}$ Due was chosen for this research paper because it has analogue $\mathrm{I} / \mathrm{O}$, the processing and storage capacity is more significant compared to other Arduinos ${ }^{\circledR}$; these characteristics are essential for HIL interaction. The communication of the DUE1 with another DUE2 is not direct, so it is necessary to add some operations so that the analog signals entering and leaving the Arduino ${ }^{\circledR}$ are compatible with each other, as seen in Fig. 5. The changes in the system frequency of area 5 (Finland) is obtained from the NPS; it is necessary to add an offset to pass the signal to the positive side; this will depend on the size of this, 1.4 is used for this model. When the analog block is used, it accepts a value uint16 but considers only the 12 most significant bits for the conversion. This block emits voltage $\left(V_{\text {out }}\right)$ on the DAC pin, such that: 


$$
V_{\text {out }}=V_{\text {ref }}\left(\frac{\text { input }}{2^{\text {Ne }}}-1\right)+V_{\text {offset }}
$$

where $\mathrm{V}_{\text {ref }}=2.2$, input $=0<$ input $<4,095, \mathrm{Ne}=12, V_{\text {offset }}=$ 0.56 . Therefore, in order to obtain the output signal in the correct ranges, it is necessary to obtain input from (1), as shown in (2).

$$
\text { input }=V_{\text {out }}\left(\frac{2^{N e}-1}{V_{\text {ref }}}\right)-V_{\text {offset }}
$$

substituting have:

$$
\text { input }=V_{\text {out }}\left(\frac{2^{12}-1}{2.2}\right)-0.56
$$

Moreover, before the Arduino ${ }^{\circledR}$ sends the signal to the outside, an offset block of 1.4 must be added to have an entirely positive signal, a gain of $\left(2^{12}-1\right) / 2.2$; also a block of data type conversion uint16, as can be seen in Fig. 5. Fig. 6 shows the output signal from DUE1, has been called analogue frequency, and the fully simulated frequency signal in Simulink ${ }^{\mathrm{TM}}$ has been called the synthetic frequency.

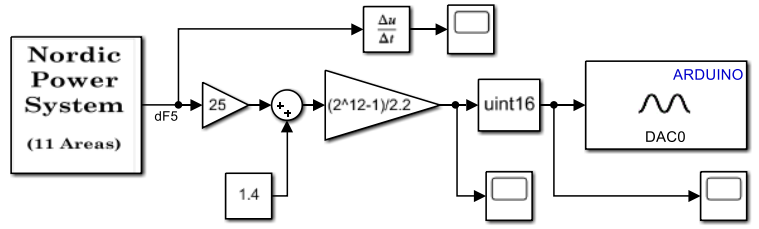

Fig. 5. Cyber implementation 1: NPS block diagram implemented in DUE1.

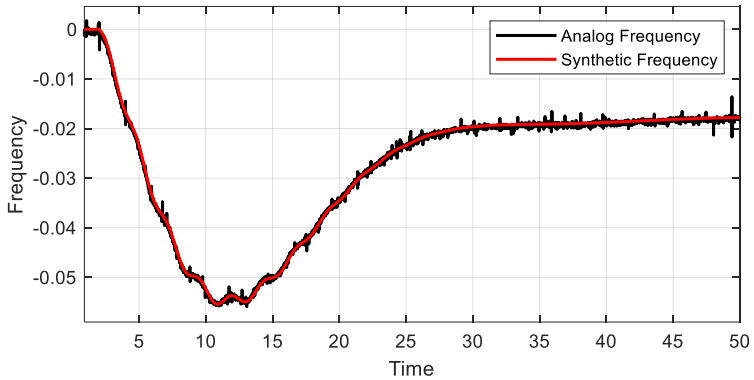

Fig. 6. Frequency signal produced by the physical system 1, implemented in DUE1.

The signal received by DUE2 in the analog input block (pin 0) defined as system frequency of area 5 comes from DUE1. If the measured voltage is equal to the analog reference voltage, the output of the block outputs 1,023. A gain of $3.3 / 1,023$ is added to perform the coupling of the analog input signal with the RoCoF calculation blocks. The offset correction is added with a constant of 1.96, as seen in Fig. 7.

\section{A. Base Case: No filtering}

This section is the beginning of the cases established to simulate the derivative methods, it was called Base Case, because filters are not included in this model.

\section{1) RoCoF 1: Incremental difference one step}

Included in this scheme is the frequency signal that comes from the NPS, area 5, the derivative block receives four inputs: frequency, the frequency with delay, time, time with delay, at the output a setting gain and the RoCoF, Fig. 7.

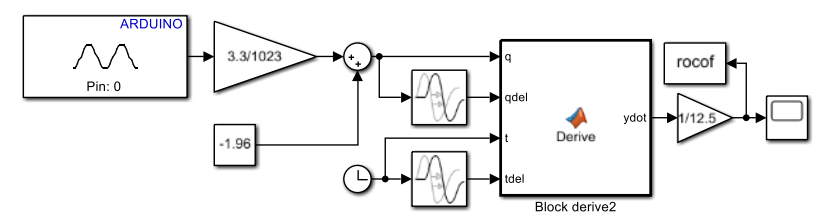

Fig. 7. Cyber Implementation 2: No filtering RoCoF 1.

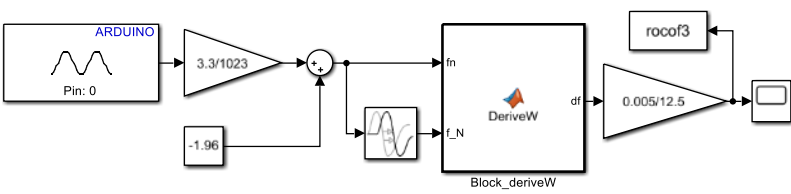

Fig. 8. Cyber Implementation 2: No filtering RoCoF 2.

2) RoCoF 2: Moving window or rolling window

In the same way as RoCoF1, this scheme includes the frequency signal that comes from the NPS, area 5, the differentiating block receives two inputs: Frequency, Frequency with delay, at the output a setting gain and the RoCoF, Fig. 8.

Fig. 9 shows an example of how the derivatives of the Base Case return the RoCoF signal without using filters, noise effects, and it is not possible to appreciate the expected signal.

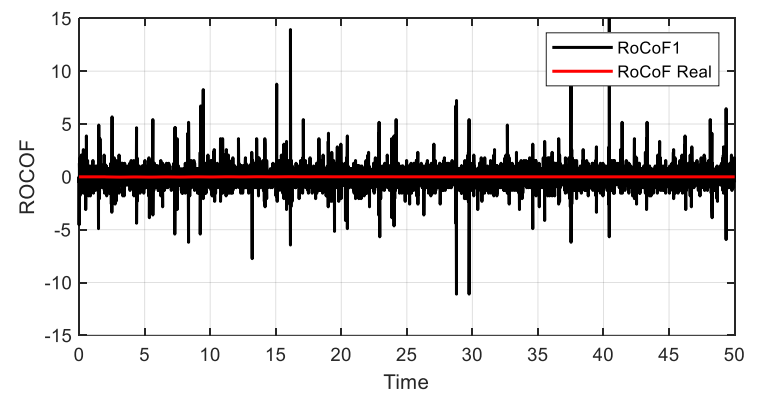

Fig. 9. RoCoF signal produced by the physical system 2: No filtering RoCoF 1.

\section{B. Case I: LPF-Frequency}

This section shows Case I, in this case, in addition to implementing the derivative, an LPF is also implemented for the frequency signal.

1) RoCoF 1: Incremental difference one step

In this scheme, an LPF is added for the frequency signal. The derivative receives 4 inputs: Frequency, Frequency with delay, time, time with delay, at the output a setting gain and the RoCoF, Fig. 10.

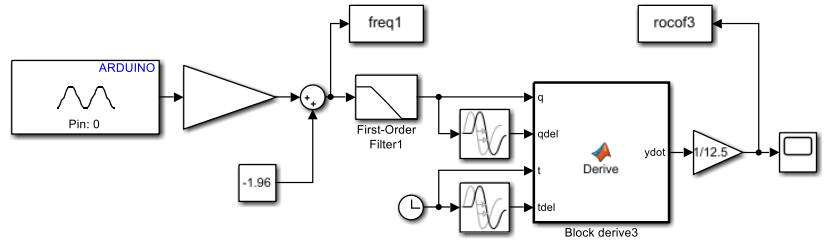

Fig. 10. Cyber Implementation 2: LPF-Frequency RoCoF 1.

Fig. 11 shows in black the RoCoF1 signal using a LPF that filters the Frequency signal. Besides, in red shows the RoCoF obtained in Simulink by the differentiating block, it has been called RoCoF Real. 


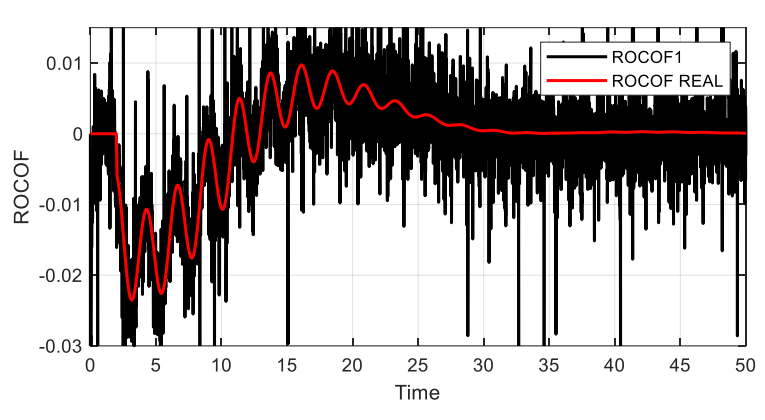

Fig. 11. RoCoF signal produced by the physical system 2: LPF-Frequency RoCoF1.

2) RoCoF2: Moving window or rolling window

In this scheme also an LPF is added for the frequency signal. The derivative receives two inputs: Frequency, the frequency with delay, at the output a setting gain and the RoCoF, Fig. 12.

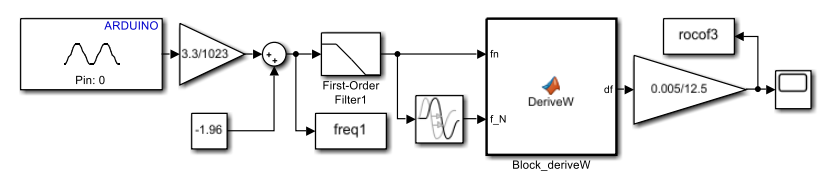

Fig. 12. LPF-Frequency RoCoF 2.

Fig. 13 shows in black the RoCoF 2 signal using an LPF that filters the frequency signal. Besides, in red shows the RoCoF Real.

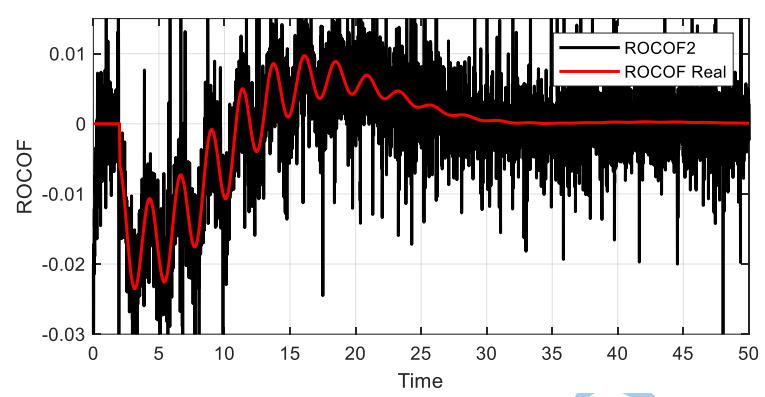

Fig. 13. RoCoF signal produced by the physical system 2: LPF-Frequency RoCoF 2.

From Fig. 11, and Fig. 13, it is possible to conclude the effect of filtering noise coming from the Arduino ${ }^{\circledR}$, unlike Fig. 10 where it is not possible to appreciate a signal, much less the behaviour, with the filter the signal is already possible to appreciate the behaviour, some of the noise has been removed.

\section{Case II: LPF-Frequency + LPF-RoCoF}

Finally, this section shows Case 2, in this case, in addition to implementing the derivative, an LPF is also implemented for the frequency signal and another LPF at the output of the derivative block.

\section{1) RoCoF1: Incremental difference one step}

In this scheme, two LPFs have been added, one for the frequency signal and the other at the output of the derivative block Fig. 14

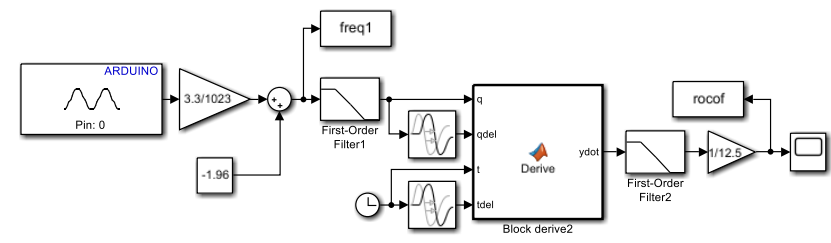

Fig. 14. Cyber Implementation 2: LPF-Frequency + LPF-RoCoF1

Fig. 15 shows in black the RoCoF 1 signal using two LPFs, for the frequency signal and the derivative block and in red shows the RoCoF Real.

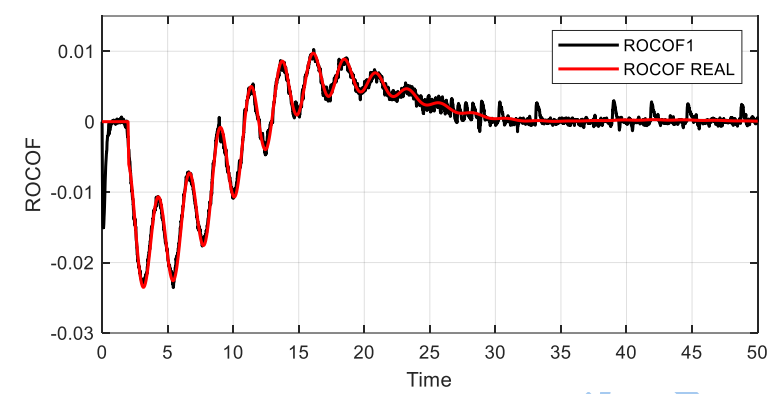

Fig. 15. RoCoF signal produced by the physical system 2: LPF-Frequency + LPF-RoCoF1

2) RoCoF2: Moving window or rolling window

In this scheme, two LPFs have been added, for the frequency signal and the output of the derived block, Fig. 16.

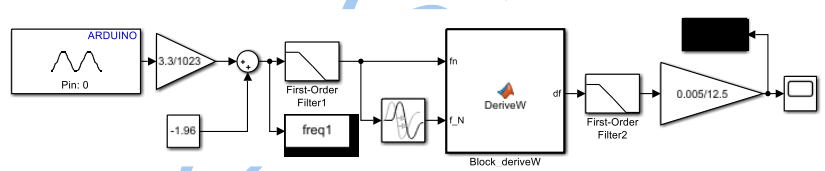

Fig. 16. LPS-Frequency + LPS-RoCoF2

Fig. 17 shows in black the RoCoF 1 signal using two LPFs, for the frequency signal and the derivative block and in red shows the RoCoF Real.

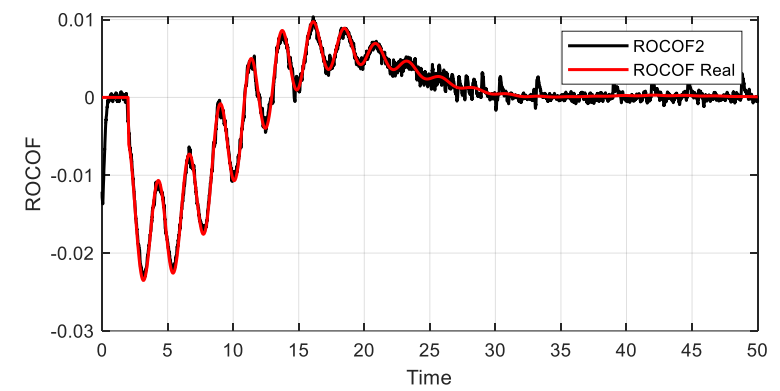

Fig. 17. RoCoF signal produced by the physical system 2: LPF -Frequency $+\mathrm{LPF}-\mathrm{RoCoF} 2$

The effect of attenuating both signals, the one from the frequency and the other from the derivative block, gives a response with a much smoother signal, compared to the signals obtained in the models without filter and with a filter.

\section{CONCLUSIONS}

This paper presented the implementation of a RoCoF calculation algorithm using low-cost HIL. For illustrative purposes, the eleven areas model of the Nordic Power System (NPS) has been used. Two methods to calculate the RoCoF are presented: Incremental difference one step and Moving window (MW) or rolling window. HIL approach is used to obtain natural noise found in the frequency signal obtained from real power devices. The low-cost HIL implementation is based on two Arduinos ${ }^{\circledR}$ in the loop, creating a cyber-physical system. It was concluded that the derivative methods worked correctly calculating the RoCoF. Using two LPF helps to clean the signal from the noise coming from the DUE1; therefore, the expected signal can be appreciated. 


\section{ACKNOWLEDGEMENT}

J. Barrios-Gomez is grateful to CONACYT-Mexico for providing a Postdoctoral research fellowship and Loughborough University, UK, for the support provided during the research visit.

\section{REFERENCES}

[1] F. Gonzalez-Longatt, F. Sanchez, and R. Leelaruji, "Unveiling the Character of the Frequency in Power Systems," in 2019 IEEE PES GTD Grand International Conference and Exposition Asia, GTD Asia 2019, 2019, pp. 57-62.

[2] M. N. Acosta, F. Gonzalez-Longatt, S. Denysiuk, and H. Strelkova, "Optimal Settings of Fast Active Power Controller: Nordic Case," in IEEE 7th International Conference "2020 IEEE ESS, ” 2020.

[3] EURAMET, "Evaluation report on the problem of ROCOF measurement in the context of actual use cases and the 'wish list' of accuracy and latency from an end-user point of view," 2018. [Online] Available: http://www.rocofmetrology.eu/wpcontent/uploads/sites/21/2018/10/ROCOF-Uses-Cases-and-TestConditions-V1.pdf.

[4] J. A. Barrios-Gomez et al., "Framework for Real-Time Simulations of Hardware in the Loop Applied to Primary Frequency Control," in IEEE 7th International Conference "2020 IEEE ESS," 2020

[5] Y. S. Wang, G. C. Shang, and T. X. He, "The PID-type fuzzy neural network control and it's application in the hydraulic turbine generators," in 2000 IEEE Power Engineering Society, Conference Proceedings, 2000, vol. 1, pp. 338-343.

[6] F. Gonzalez-Longatt, J. Rueda, and E. Vazquez, Effect of Fast Acting Power Controller of Battery Energy Storage Systems in the Underfrequency Load Shedding Scheme. 2018.

[7] C. F. Ten and P. A. Crossley, "Evaluation of ROCOF relay performances on networks with distributed generation," in IET Conference Publications, 2008, no. 536 CP, pp. 522-527.

[8] A. Beddoes, P. Thomas, and M. Gosden, "Loss of Mains protection relay performances when subjected to network disturbances / events," in IEE Conference Publication, 2005, vol. 4, no. 2005-11034, pp. 193-197.

[9] A. Perilla, J. L. Rueda Torres, S. Papadakis, E. Rakhshani, M. van der Meijden, and F. Gonzalez-Longatt, "Power-Angle Modulation Controller to Support Transient Stability of Power Systems Dominated by Power Electronic Interfaced Wind Generation," Energies, vol. 13, no. 12, p. 3178, Jun. 2020

[10]R. Bugdał, A. Dyśko, G. M. Burt, and J. R. Mcdonald, "Performance analysis of the ROCOF and Vector Shift methods using a dynamic protection modelling approach," Response, pp. 139-144, 2006.

[11]C. G. Bright, "COROCOF: Comparison Of Rate Of Change Of Frequency protection. A solution to the detection of loss of mains," in 2001 Seventh International Conference on Developments in Power System Protection (IEE), 2005, pp. 70-73.

[12]D. Carrión, A. Ayo, and J. W. González, "Alternative Under Frecuency Load Disconection Methodology Based on Semi-Adaptative Model," Enfoque UTE, vol. 11, no. 1, pp. 96-107, Jan. 2020

[13] W. Freitas, W. Xu, C. M. Affonso, and Z. Huang, "Comparative analysis between ROCOF and vector surge relays for distributed generation applications," IEEE Trans. Power Deliv., vol. 20, no. 2 II, pp. 13151324, 2005.

[14] M. Grebla, J. Yellajosula, and H. C. Hoidalen, "Adaptive Frequency Estimation Method for ROCOF Islanding Detection Relay," IEEE Trans. Power Deliv., pp. 1-1, 2019.

[15]F. S. Gorostiza and F. Gonzalez-Longatt, "Deep Reinforcement Learning-Based Controller for SOC Management of Multi-Electrical Energy Storage System," IEEE Trans. Smart Grid, vol. 3053, no. c, pp. $1-1,2020$.

[16] A. Peña Asensio, F. Gonzalez-Longatt, S. Arnaltes, and J. L. RodríguezAmenedo, "Analysis of the Converter Synchronizing Method for the Contribution of Battery Energy Storage Systems to Inertia Emulation," Energies, vol. 13, no. 6, p. 1478, Mar. 2020

[17]D. Doheny and M. Conlon, "Investigation into the local nature of rate of change of frequency in electrical power systems," in 2017 52nd International Universities Power Engineering Conference, UPEC 2017, 2017, vol. 2017-Janua, pp. 1-6.

[18]F. Sanchez and F. Gonzalez-Longatt, "Optimization of Frequency Controller Parameters of a BESS by considering Rate of Change Constraints," in 2019 IEEE Milano PowerTech, Powertech 2019, 2019.

[19]P. M. (Paul M. . Anderson, A. A. (Abdel-A. A. . Fouad, and Institute of Electrical and Electronics Engineers., Power system control and stability. .

[20] O. Dudurych and M. Conlon, "Impact of reduced system inertia as a result of higher penetration levels of wind generation," in Proceedings of the Universities Power Engineering Conference, 2014, pp. 1-6.

[21]P. J. Olver, "Introduction to Partial Differential Equations," in Pure and Applied Mathematics, vol. 1, no. C, Springer International Publishing, 1949, pp. 32-62.

[22]R. L. Burden and J. D. Faires, Numerical Analysis, Fourth. Boston: \{PWS-Kent Publishing Company, 1989.

[23]D. Moskau, "Application of real time digital filters in NMR spectroscopy," Concepts Magn. Reson. Part B Magn. Reson. Eng., vol. 15 , no. 2, pp. 164-176, Jun. 2002.

[24]S. W. Smith, The Scientist and Engineer's Guide to Digital Signal Processing. USA: California Technical Publishing, 1997.

[25]G. Chown, J. Wright, R. van Heerden, and M. Coker, "System inertia and Rate of Change of Frequency (RoCoF) with increasing nonsynchronous renewable energy penetration, Innovation in the Power Systems industry," CIGRE Sci. -Engineering, vol. 11, no. June, pp. 1134, Nov. 2018.

[26]S. ROGERS, "Analog and digital filter design using C," Control Eng. Pract., vol. 4, no. 8, pp. 1191-1192, 1996.

[27] "Nord Pool Spot," About us. [Online]. Available: https://www.nordpoolgroup.com/About-us/. [Accessed: 17-Dec-2015].

[28] "Statnett," Kraftsystemet akkurat $n^{\circ}$ a (Norwegian) [The power system right now]. [Online]. Available: https://www.statnett.no/Drift-ogmarked/. [Accessed: 24-Nov-2015].

[29]K. G. Andersen, "Area Based Secondary Frequency Control in the Nordic Power System," no. June, p. 123, 2016.

[30]MathWorks, "Arduino Support from Simulink," 2016. [Online]. Available: https://uk.mathworks.com/hardware-support/arduinosimulink.html. 